Published in final edited form as:

Curr Opin Virol. 2016 December ; 21: 109-113. doi:10.1016/j.coviro.2016.08.017.

\title{
Adenovirus sensing by the immune system
}

\author{
Svetlana Atashevaa,\# and Dmitry Shayakhmetov ${ }^{a, b, \#}$ \\ aDepartment of Pediatrics, Division of Rheumatology, Emory Children's Center for Transplantation \\ and Immune Mediated Disorders, Department of Medicine, 1760 Haygood Drive, Emory \\ University, Atlanta, GA 30322, USA \\ bLowance Center for Human Immunology, Emory Children's Center for Transplantation and \\ Immune Mediated Disorders, Department of Medicine, 1760 Haygood Drive, Emory University, \\ Atlanta, GA 30322, USA
}

\section{Abstract}

The host immune system developed multiple ways for recognition of viral pathogens. Upon disseminated adenovirus infection, the immune system senses adenovirus invasion from the moment it enters the bloodstream. The soluble blood factors, FX, antibodies, and complement, can bind and activate plethora of host-protective immune responses. Adenovirus binding to the cellular $\beta 3$ integrin and endosomal membrane rupture trigger activation of IL-1a/IL-1R1 proinflammatory cascade leading to attraction of cytotoxic immune cells to the site of infection. Upon cell entry, adenovirus exposes its DNA genome in the cytoplasm and triggers DNA sensors signaling. Even when inside the nucleus, the specialized cellular machinery that recognizes the double-strand DNA breaks become activated and triggers viral DNA replication arrest. Thus, the host employs very diverse mechanisms to prevent viral dissemination.

\section{Introduction}

Prompt recognition of a viral invasion and timely activation of antiviral mechanisms are crucial survival factors for the host. The diversity of viruses and the continuous threat they pose, forced hosts to develop multiple ways of recognizing "non-self" virus determinants. The pathogen-sensing mechanisms in the host target every step of viral infection ensuring detection and elimination of the pathogen by the immune system. Activation of the immune system by Adenovirus (Ad) triggers multiple defense mechanisms aimed at clearing the viral pathogen. The first steps of the immune activation include: (i) activation of a systemic proinflammatory state, (ii) attracting cytotoxic immune cell populations to the sites of infection to eliminate virus-containing cells, and (iii) alarming neighboring uninfected cells of viral infection. The systemic proinflammatory state is achieved by the release of proinflammatory cytokines IL-6, tumor necrosis factor alpha (TNFa), and IL-1 $\beta$ into the bloodstream [1]. Macrophages (MF) in different organs represent the first cellular line of defense that traps

\footnotetext{
\#Corresponding authors, svetlana.atasheva@emory.edu or dmitryshay@emory.eduPhone: 1-404-727-4038.

Publisher's Disclaimer: This is a PDF file of an unedited manuscript that has been accepted for publication. As a service to our customers we are providing this early version of the manuscript. The manuscript will undergo copyediting, typesetting, and review of the resulting proof before it is published in its final citable form. Please note that during the production process errors may be discovered which could affect the content, and all legal disclaimers that apply to the journal pertain.
} 
incoming Ad, thus withdrawing it from the bloodstream [2-6]. Next, infected MF release different cytokines and chemokines that function as chemoattractants for the cytotoxic immune cells. The IL-1a/IL-1R1 pathway is critical for the activation of incoming cytotoxic cells to eliminate infected cells and prevent virus spread[7]. To alarm neighboring noninfected cell of a viral infection, Ad infected cells release type I interferons (IFN), IFNa and IFN $\beta$ [8]. These cytokines activate the expression of numerous effector antiviral genes in surrounding cells, preparing uninfected cells to enter an antiviral state. Given the complexity of the immune response to viral infection it is quite difficult to untangle the signaling pathways in different cell populations in the host and determine particular roles of pathways in viral clearance. This review focuses on the recent data of the mechanisms that hosts employ for sensing Ad vector and signaling pathways activated by Ad vector infection.

\section{Blood factor signaling}

Sensing Ad vectors by the host occurs at all stages of viral invasion starting from the moment it enters the bloodstream. Upon intravascular delivery, the Ad vector interacts with multiple blood factors, including coagulation factor X (FX), neutralizing and natural antibody ( $\mathrm{Ab}$ ), and complement components $\mathrm{C} 3$ and $\mathrm{C} 4$ [4, 9-11]. The host machinery detects the interactions of Ad with blood factors and activates the immune system. Binding of FX to the Ad hexon, one of the major capsid proteins, results in nuclear factor kappa B (NFkB)-dependent transcriptional activation of IL-1 $\beta$ cytokine in the spleen [9]. The mechanism of detection of Ad-FX complex in vivo requires toll-like receptor 4 (TLR4) signaling and functional activity of TLR4 adaptors MyD88, TRAF6, and TRIF. In support of this, a mutant Ad5 vector containing a single mutation in the hexon protein T425A that ablates binding to the FX fails to elicit IL-1 $\beta$ transcription in vivo [9]. Additionally, Ad subtypes that are naturally incapable of FX binding elicit a diminished multitude of cytokines compared to Ad subtypes that bind the FX [9]. The TLR4 signaling was also shown to be important for the ability to attract and retain polymorphonuclear leukocytes (PMNs) in the marginal zone of the spleen. As a result, mice deficient in TLR4 failed to clear the virus [9]. In addition, other blood soluble factors besides FX have been shown to play a role in sensing Ad. McEwan et al showed that the tripartite motif containing 21(TRIM21), an intracellular antibody receptor, can recognize Ab-Ad complexes and trigger activity [11]. TRIM21 was able to recognize intracellular Ab and activate the synthesis of an unanchored Lys63 polyubiqitin chain, which, in turn, activated, Activator protein 1 (AP1), and interferon regulating factor (IRF) signaling pathways. As a result, TRIM21-deficient MEFs had significantly (8-1000 fold) lower expression of a variety of pro-inflammatory cytokines and chemokines (IL- 6, CXCL10, CCL2, CCL4, TNFa, and IFN $\beta$ ) in response to treatment with Ad-Ab complex. The activation of signaling pathways by Abs in the cytosol was independent of FcR or pattern recognition receptors (PRRs), but dependent on transforming growth factor beta-activated kinase 1 (TAK1) and signaling [11]. Interestingly, no difference was found in activating abilities between IgG or IgM. Moreover, transfection of Abs bound to the latex beads was also able to activate signaling in MEFs [11]. Another report implicating a blood factor, complement component $\mathrm{C} 3$, in Ad sensing came from the same group. Tam et al showed that C3 in complex with Ad can be detected in the cytosol by the unknown cytoplasmic receptor [12]. The intracellular sensing of the complement 
activated inflammatory cascades dependent on mitochondrial activating signaling protein (MAVS) and TRAF signaling. Interestingly, cells lacking complement receptors on the surface were able to activate the signaling cascades only after C3-Ad complex is located in the cytoplasm, whereas cells that have activating complement receptors CR1, CR3, or CR4 were able to sense the complex on the cellular surface and activate signaling [12]. The studies implicating the intracellular sensing of complement components and antibodies in the activation of the immune system have been performed exclusively in tissue culture and their applicability in the in vivo settings require further elucidation. In summary, the blood components FX, C3, and natural antibodies represent very ancient pathogen detection system and their relocalization into the cytosol triggers a nonspecific systemic proinflammatory response.

\section{Macrophages signaling}

The next step of pathogen recognition occurs when the virus attaches to the receptor on the cellular surface and enters the cell. The fiber protein of the Ad capsid mediates the primary receptor attachment of the virus. The very distal fiber domain, fiber knob, binds to the primary species-specific receptor activating the virus entry to the cell [13]. The secondary attachment is mediated by the RGD loops located on the penton protein of Ad capsid. The RGD motif on the penton functions as a ligand for $\alpha 3 \beta 1, \alpha 5 \beta 1, \alpha v \beta 1, \alpha v \beta 3$, and $\alpha v \beta 5$ integrins on the surface of the cell [14]. In the organs, residential MFs are the first line of the cells that interacts with the incoming Ad vector. In the spleen $\mathrm{MARCO}^{+} \mathrm{M \Phi s} \mathrm{trap} \mathrm{the} \mathrm{virus}$ from the bloodstream and release IL-1a thus activating IL-1R1 dependent innate immune response [15]. The engagement of $\beta 3$ integrins during Ad entry was found to be critical for IL-1 $\alpha$ expression. The mice deficient in $\beta 3$ integrin did not activate full immune response upon Ad vector injection, and reciprocally, Ad vector with deleted RGD motif was unable to activate innate immune response [16]. The $\mathrm{Ad}$ ts 1 mutant that binds to the $\beta 3$ integrins but is unable to rupture the endosome was also very weak innate immune system inducer [15]. However, the ts1 mutant was able to induce the expression of IL-1a mRNA, but failed to produce functionally active IL-1a. These data suggest that engagement of $\beta 3$ integrin on the cellular surface triggers activation of IL-1a mRNA transcription, but for cytokine production the second signal, such as an endosomal rupture is required. Furthermore, IL-1a signaling through the IL-1R1 and activation of expression CXCL1 and CXCL2 chemokines leads to attraction of PMNs to the sites of infection [2, 7]. Interestingly, that complement component C3 cooperated for the recruitment of PMNs to the spleen. Treatment of mice with complement inhibitory proteins resulted in partial redistribution of PMNs from the spleen marginal zone, while the same treatment of IL-1a-deficient mice completely ablated PMNs localization in the marginal zone. In summary, in the spleen the activation of the key IL-1a/ IL-1R1 proinflammatory pathway is dependent on $\beta 3$ integrin- Ad penton RGD interaction, as well as on the endosomal membrane rupture [2]. The IL-1a signaling cooperates with complement $\mathrm{C} 3$ for attracting and retaining PMNs in the spleen marginal zone that kill and eliminate infected MFs.

In the liver the first cells that encounter the incoming vector are Kupffer cells, residential liver MФs. Kupffer cells trap the virus from the bloodstream and in the attempt to eliminate viral burden by undergoing a suicidal necrotic cell death [17, 18]. The Kupffer cell death 
occurs within the first hour post Ad vector injection and does not require signaling of any known mediators of necrotic cell death $[3,19]$. However, Kupffer cells in mice deficient in IRF3 were unable to trigger the necrotic cells death, subsequently leading to significantly lower amount of proinflammatory cytokines and chemokines in the plasma and higher viral load at $24 \mathrm{~h}$ post injection in IRF3 ${ }^{-/-}$mice than in wt animals [3]. The absence of the MФ death was also observed in the wt mice injected with the ts 1 mutant of Ad that is incapable of escaping the endosomes. Interestingly, the deficiency in the upstream activators of IRF3 (MAVS, stimulator of interferon genes (STING), and DNA-dependent activator of interferon-regulatory factors (DAI)) did not prevent Kupffer cell death suggesting an alternative mechanism for sensing Ad. Thus, the Kupffer cells in the liver sense Ad through the membrane rupture and an unknown receptor leading to IRF3-dependent necrotic cell death [3].

Activation of the NLR family pyrin domain containing 3 (NLRP3) inflammasome pathway, another necrotic cell death pathway, upon Ad infection was shown by Muruve et al in human THP1 cells that release mature IL- $1 \beta$ in response to infection with Ad5 or Ad3 [20]. The IL-1 $\beta$ release was dependent on the formation of the NALP3 inflammasome and functioning of its adaptor apoptosis-associated speck-like protein containing a CARD (ASC) [21]. The experiments showed that the non-replicating Ad, helper-dependent Ad, or vectors with ablated receptor were able to activate expression of IL- $1 \beta$ to the same extent as wt Ad. However, empty capsids or naked DNA failed to induce IL-1 $\beta$ expression [20]. Although, DNA delivered into the cytoplasm with liposomes was able to trigger the inflammasome activation, suggesting the role of an intracellular viral DNA sensor. Though, no difference in IL-1 $\beta$ expression was observed in peritoneal MФs derived from the mice deficient in TLR9, an endosomal DNA sensor, or its adaptor MyD88. The authors suggest that the cells sense Ad vector DNA by not yet defined DNA sensor.

In contrast, Cerullo et al showed that isolated peritoneal MФs secrete IL-6 in response to infection with helper-dependent $\mathrm{Ad}$, while $\mathrm{M} \Phi$ s derived from mice having nonfunctional

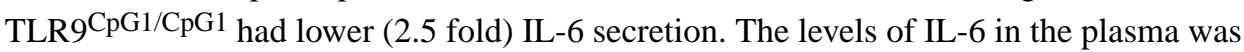

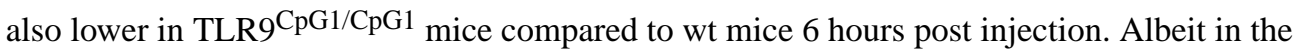
TLR9 deficient mice the IL-6 levels were still high, suggesting an alternative TLR9independent sensing mechanisms [22]. The role of TLR9, was shown in another study in human monocytic cell line THP1. Ad-dependent NLRP3 inflammasome activation and IL-1 $\beta$ expression in THP1 cells required activation of production of reactive oxygen species (ROS) and TLR9 signaling [23]. Moreover, the ROS production was independent of TLR9 signaling but was induced by the Ad-dependent membrane rupture, release of cathepsin B, and destabilization of the mitochondrial membranes [24].

To carry on the downstream activation cascades TLR9 signaling requires its adaptor MyD88. MyD88 also serves as an adaptor to other TLRs [25]. Mice lacking MyD88 were unable to activate and failed to produce IL-6, CXCL1, CCL2, CCL4 cytokines and chemokines in the liver after i.v. Ad vector administration [26]. The IL-6 and CCL2 expression dependent on TLR9 activation, while CXCL1 and CCL4 expression did nor depend on neither TLR2 nor TLR9 signaling in the liver. Moreover, mice treated with chlodronate liposomes, thus lacking Kupffer cells in the liver, were unable to produce CCL2 and CXCL1, while IL-6 and 
CCL4 levels were not significantly lower, suggesting the role of non Kupffer cells in MyD88-dependent sensing of Ad [26].

\section{Type I IFN activation}

The type I IFN signaling mediates the activation of an antiviral state in notyet- infected cells surrounding the infection site. TLR9 signaling via MyD88 adaptor was absolutely required for IFNa production ex vivo in isolated splenic plasmacytoid dendritic cells (pDCs) [27]. The non pDC cells like Kupffer cells, conventional DCs, or peritoneal MFs did not require TLR9 signaling for IFNa activation. In vivo data supported a multiple factor sensing mechanisms, TLR9 deficient mice had lower level of serum IFNa than wt mice after Ad vector administration, but still at detectable levels, suggesting an alternative, TLR9 independent mechanism of IFN activation [27]. In non pDCs the IFNa induction was triggered by the cytosolic recognition of DNA and in MyD88 independent manner. In another line of studies activation of IFN $\beta$ induction in response to Ad vector administration required viral entry, endosomal escape, and exposure of viral DNA in the cytoplasm [28]. The sensing of the Ad DNA in the cytoplasm occurred by the cytosolic DNA sensor cyclic GMP-AMP synthase (cGAS) [29]. This molecule senses DNA in the cytoplasm, changes conformation, homodimerizes and synthesizes a messenger molecule, 2'-3' cyclic guanine adenine monophosphate (cGAMP) [30]. cGAMP binds to the adaptor protein STING, leading to activation of tank-binding kinase 1 (TBK1) and IRF3 [30, 31]. Bone marrowderived МФs (BMMФ) deficient in cGAS/STING have almost unresponsive phenotype to Ad infection, suggesting the major role of this pathway in ВММФs. In contrast, BMDC derived from mice lacking cGAS/STING signaling have residual activity suggesting involvement of other signaling pathways [29]. In the liver, the absence of cGAS/STING pathway resulted in significantly lower activation of IFN $\beta$ and IFN-stimulated genes ISG15 and ISG54. Additionally to activating IFN type I response, in the absence of the cGAS/ STING pathway, IL-6 and TNFa, and CXCL1 were diminished in mouse serum $6 \mathrm{~h}$ postinfection with Ad vector [29]. Taken together, the multiple reports show that different cell populations are capable to sense Ad vector and activate type I IFN production by exploiting different means, however the major trigger for IFN activation is sensing viral DNA in the cell.

\section{Nuclear sensing of adenovirus}

The host DNA repair machinery has also means to distinguish viral DNA versus cellular DNA in the nucleus. Ad DNA replicates in the nucleus and produces significant amounts of the double-strand DNA ends, that are sensed by the cellular DNA damage response machinery, namely MRE11/RAD50/NBS1 (MRN) complex. This complex binds to the dsDNA breaks and activates DNA damage response that results in replication arrest. By the unknown mechanism the MRN complex can activate only a local DNA damage response arresting only replication of viral DNA without activating a global arrest [32]. Ads encode two proteins E1B-55K and E4-ORF3 that inactivate MRN complex preventing DNA damage response activation. These proteins play redundant role in MRN inactivation, in the absence of both proteins MRN binding to the viral DNA and activation of DNA damage response leads to inhibition of the viral DNA replication [32]. Additionally, Ad protein VII in the 
nucleus binds and sequesters high-mobility-group B protein 1 (HMGB1) that functions as an immune danger signal if released from the cell. However, the mechanism of sensing the Ad infection and release of HMBG1 is unknown [33].

In summary, the Ad sensing mechanisms of the host are highly variable and target all stages of viral replication. Ad vector in the bloodstream is interacting with blood factors such as $\mathrm{FX}, \mathrm{Ab}$, and complement, these interaction alert the host of the viral presence by activating $\mathrm{NF} \times \mathrm{B}$ - dependent signaling cascades, resulting in a general proinflammatory state, mediated by IL- 6 , TNFa, and IL- $1 \beta$ cytokines. In different organs the MFs trap the virus from the bloodstream and activate proinflammatory signaling leading to PMN attraction and retention in the infection sites. Different populations of cells have different means of virus sensing, but generally the viral determinants include viral DNA in the cytosol, engaging $\beta 3$ integrins, and endosomal membrane rupture. Moreover cells can sense replicating viral DNA and inhibit viral replication by activating the DNA damage response. These multiple mechanisms are all aimed at alerting the host of the viral invasion, eliminating the virus infected cells and activating an antiviral state.

\section{Acknowledgments}

This work was supported by the funds from the US National Institutes of Health grants AI065429, AI107960, AI126816, and funds from the Children's Healthcare of Atlanta Research Trust to D.M.S.

\section{References}

1. Mistchenko AS, et al. Cytokines in adenoviral disease in children: association of interleukin-6, interleukin-8, and tumor necrosis factor alpha levels with clinical outcome. J Pediatr. 1994; 124(5 Pt 1):714-720. [PubMed: 8176557]

2. Di Paolo NC, et al. IL-1alpha and complement cooperate in triggering local neutrophilic inflammation in response to adenovirus and eliminating virus-containing cells. PLoS Pathog. 2014; 10(3):e1004035. [PubMed: 24651866]

3. Di Paolo NC, et al. The transcription factor IRF3 triggers "defensive suicide" necrosis in response to viral and bacterial pathogens. Cell Rep. 2013; 3(6):1840-1846. [PubMed: 23770239] The report shows an example of macrophage sensing and signaling in response to adenovirus infection. Upon intravascular adenovirus delivery Kupffer cells in the liver undergo necrotic cell death. The autors showed that this type of death is not dependent on any known cell death mediators and functions as an effective innate immune mechanism to protect the host from viral infection.

4. Khare R, et al. Circulating antibodies and macrophages as modulators of adenovirus pharmacology. J Virol. 2013; 87(7):3678-3686. [PubMed: 23325678]

5. $\mathrm{Xu} \mathrm{Z}$, et al. Clearance of adenovirus by Kupffer cells is mediated by scavenger receptors, natural antibodies, and complement. J Virol. 2008; 82(23):11705-11713. [PubMed: 18815305]

6. Smith JS, et al. Unexpected pulmonary uptake of adenovirus vectors in animals with chronic liver disease. Gene Ther. 2004; 11(5):431-438. [PubMed: 14973536]

7. Kolaczkowska E, Kubes P. Neutrophil recruitment and function in health and inflammation. Nat Rev Immunol. 2013; 13(3):159-175. [PubMed: 23435331]

8. Randall RE, Goodbourn S. Interferons and viruses: an interplay between induction, signalling, antiviral responses and virus countermeasures. J Gen Virol. 2008; 89(Pt 1):1-47. [PubMed: 18089727]

9. Doronin $\mathrm{K}$, et al. Coagulation factor $\mathrm{X}$ activates innate immunity to human species $\mathrm{C}$ adenovirus. Science. 2012; 338(6108):795-798. [PubMed: 23019612]

10. Waddington SN, et al. Adenovirus serotype 5 hexon mediates liver gene transfer. Cell. 2008; 132(3):397-409. [PubMed: 18267072] 
11. McEwan WA, et al. Intracellular antibody-bound pathogens stimulate immune signaling via the $\mathrm{Fc}$ receptor TRIM21. Nat Immunol. 2013; 14(4):327-336. [PubMed: 23455675]

12. Tam JC, et al. Intracellular sensing of complement $\mathrm{C} 3$ activates cell autonomous immunity. Science. 2014; 345(6201):1256070. [PubMed: 25190799] This report is an example of a blood factor signaling event. The authors showed that the deposition of the complement component C3 on the viral particle and subsequent relocalization into the cytosol triggers an antiviral state in the cells.

13. Nemerow GR, Stewart PL, Reddy VS. Structure of human adenovirus. Curr Opin Virol. 2012; 2(2): 115-121. [PubMed: 22482707]

14. Nemerow GR, Stewart PL. Role of alpha(v) integrins in adenovirus cell entry and gene delivery. Microbiol Mol Biol Rev. 1999; 63(3):725-734. [PubMed: 10477314]

15. Di Paolo NC, et al. Virus binding to a plasma membrane receptor triggers interleukin-1 alphamediated proinflammatory macrophage response in vivo. Immunity. 2009; 31(1):110-121. [PubMed: 19576795]

16. Koizumi N, et al. Modified adenoviral vectors ablated for coxsackievirus-adenovirus receptor, alphav integrin, and heparan sulfate binding reduce in vivo tissue transduction and toxicity. Hum Gene Ther. 2006; 17(3):264-279. [PubMed: 16544976]

17. Lyons $\mathrm{M}$, et al. Adenovirus type 5 interactions with human blood cells may compromise systemic delivery. Mol Ther. 2006; 14(1):118-128. [PubMed: 16580883]

18. Tao N, et al. Sequestration of adenoviral vector by Kupffer cells leads to a nonlinear dose response of transduction in liver. Mol Ther. 2001; 3(1):28-35. [PubMed: 11162308]

19. Shashkova EV, et al. Macrophage depletion combined with anticoagulant therapy increases therapeutic window of systemic treatment with oncolytic adenovirus. Cancer Res. 2008; 68(14): 5896-5904. [PubMed: 18632644]

20. Muruve DA, et al. The inflammasome recognizes cytosolic microbial and host DNA and triggers an innate immune response. Nature. 2008; 452(7183):103-107. [PubMed: 18288107]

21. Franchi L, et al. Intracellular NOD-like receptors in innate immunity, infection and disease. Cell Microbiol. 2008; 10(1):1-8. [PubMed: 17944960]

22. Cerullo V, et al. Toll-like receptor 9 triggers an innate immune response to helper-dependent adenoviral vectors. Mol Ther. 2007; 15(2):378-385. [PubMed: 17235317]

23. Barlan AU, et al. Adenovirus membrane penetration activates the NLRP3 inflammasome. J Virol. 2011; 85(1):146-155. [PubMed: 20980503]

24. McGuire KA, et al. Adenovirus type 5 rupture of lysosomes leads to cathepsin B-dependent mitochondrial stress and production of reactive oxygen species. J Virol. 2011; 85(20):1080610813. [PubMed: 21835790]

25. O'Neill LA, Bowie AG. The family of five: TIR-domain-containing adaptors in Toll-like receptor signalling. Nat Rev Immunol. 2007; 7(5):353-364. [PubMed: 17457343]

26. Appledorn DM, et al. Adenovirus vector-induced innate inflammatory mediators, MAPK signaling, as well as adaptive immune responses are dependent upon both TLR2 and TLR9 in vivo. J Immunol. 2008; 181(3):2134-2144. [PubMed: 18641352]

27. Zhu J, Huang X, Yang Y. Innate immune response to adenoviral vectors is mediated by both Tolllike receptor-dependent and -independent pathways. J Virol. 2007; 81(7):3170-3180. [PubMed: 17229689]

28. Nociari M, et al. Sensing infection by adenovirus: Toll-like receptor-independent viral DNA recognition signals activation of the interferon regulatory factor 3 master regulator. J Virol. 2007; 81(8):4145-4157. [PubMed: 17251283]

29. Anghelina D, Lam E, Falck-Pedersen E. Diminished Innate Antiviral Response to Adenovirus Vectors in cGAS/STING-Deficient Mice Minimally Impacts Adaptive Immunity. J Virol. 2016; 90(13):5915-5927. [PubMed: 27076643] The manuscript shows the importance of viral genome signaling during adenovirus infection in vivo. The authors compared the proinflammatory response to adenovirus infection in antigen presenting cells derived from mice deficient in cGAS, STING, or IRF3.

30. Gao P, et al. Cyclic $\left[\mathrm{G}\left(2^{\prime}, 5^{\prime}\right) \mathrm{pA}\left(3^{\prime}, 5^{\prime}\right) \mathrm{p}\right]$ is the metazoan second messenger produced by DNAactivated cyclic GMP-AMP synthase. Cell. 2013; 153(5):1094-1107. [PubMed: 23647843] 
31. Tanaka Y, Chen ZJ. STING specifies IRF3 phosphorylation by TBK1 in the cytosolic DNA signaling pathway. Sci Signal. 2012; 5(214):ra20. [PubMed: 22394562]

32. Shah GA, O'Shea CC. Viral and Cellular Genomes Activate Distinct DNA Damage Responses. Cell. 2015; 162(5):987-1002. [PubMed: 26317467] The manuscript describes an elegant anti-viral mechanism targeting viral replication mediated by local DNA damage response machinery.

33. Avgousti DC, et al. A core viral protein binds host nucleosomes to sequester immune danger signals. Nature. 2016; 535(7610):173-177. [PubMed: 27362237] 
Highlights

- $\quad$ Soluble blood factors interact with adenovirus capsid and activate NFkB-dependent signaling

- $\quad$ Macrophages activate proinflammatory response by sensing viral entry and intracellular viral DNA

- $\quad$ DNA damage response machinery senses double-strand DNA ends of adenovirus genome and triggers viral replication arrest 